\title{
CYTOGENETIC STUDY ON WORKMEN OCCUPATIONALLY EXPOSED TO PESTICIDES
}

\author{
Omari YI*
}

*Corresponding Author: Yousif I. Omari, LFIBA, Department of Biological Sciences, Faculty of Science, University of Jordan, Amman, Jordan; Tel: +962-6-5355000, Ext. 22210; Fax:+9626-5348932, E-mail: yomari@ju.edu.jo

\begin{abstract}
A cytogenetic study was performed on 40 workmen who were exposed to the pesticides malathion and chlorpyrifos and on 30 healthy males who had not been so exposed. The exposed workers had a consistent increase in chromosome abnormalities including chromatid gap, chromatid break, isochromatid break, dicentric and ring chromosomes, as determined by the standard chromosome aberration assay, when compared to the control group. The incidence was significantly higher in exposed smokers than that for exposed non smokers and than that for the unexposed controls as well. These findings provide further evidence for the intrinsic mutagenic activity of the pesticides studied.

Key words: Chromosomal aberrations; Organophosphate; Smokers

\section{INTRODUCTION}

Application of pesticides in agriculture is still the most effective and accepted means for production of better crops. Their widespread application shows deleterious effects on the environment and human life in different ways. It is usually connected with serious problems of pollution and health hazards [1-7]. A subtle danger from wide scale use of

Department of Biological Sciences, Faculty of Science, University of Jordan, Amman, Jordan these chemicals may be mutagenesis and toxicity to a broad range of organisms, either by direct exposure or by their ingestion through the food chain [811]. Whereas several studies have reported adverse effects of pesticides on chromosomes in a laboratory test system [9,12-17], few have been done in populations occupationally exposed to pesticides $[18,19]$. In studies of chromosome aberrations in workers occupationally exposed to pesticides both positive [3,18-21] and negative findings [22,23] have been reported. This prompted one to study the cytogenetic effects in agricultural workers occupationally exposed to pesticides in Jordan, most of whom are not protected during their use in the fields.

\section{MATERIALS AND METHODS}

The study included 40 healthy male agricultural workers (age range 24-29 years) with a mean age of 26.3 years who were exposed to the pesticides malathion and chlorpyrifos with varied durations of exposure (2-5 years) in Jordan. Thirty unexposed healthy males of the same communities (age range 23-28 years) with a mean age of 26.10 years who had no occupational contact with pesticides, were used as a control group. Twenty of the 40 workers and 16 of the 30 controls were smokers. All the participants in the study completed a questionnaire about their medical and occupational history. Any of the individuals who had been exposed to any agent known to interfere with the results, such as expo- 
sure to X-ray, to viral infection, or to drugs within a period of 3-4 months preceding the blood sampling was excluded.

Peripheral blood samples were collected by the classical method and sent coded to the Genetics Laboratory (Department of Biological Sciences, University of Jordan, Amman, Jordan) for cytogenetic analysis. Lymphocyte cultures were performed according to Hungerford [24], with minor modifications. The lymphocytes were cultured in RPMI 1640, supplemented with $20 \%$ fetal calf serum, penicillin-streptomycin solution and $0.2 \mathrm{~mL}$ phytohemagglutinin. Incubation was at $37^{\circ} \mathrm{C}$ for 48 hours and, during the last 2 hours, colcemid $(0.1 \mu \mathrm{g} /$ $\mathrm{ml}$ ) was added to produce mitotic arrest. The cells were then treated with hypotonic potassium chloride $(0.075 \mathrm{M} \mathrm{KCI})$ and fixed with glacial acetic acid in methanol (1:3). Air-dried slides were stained with $10 \%$ Giemsa. A total of 200 cells per individual were examined. Only cells with complete chromosome number were scored for chromosomal aberrations which were classified and recorded as recommended by the World Health Organization [25]. The data obtained were statistically analyzed by means of the $t$-test.

\section{RESULTS}

The cytogenetic analysis of structural chromosome aberrations was performed in 8,000 cells from the 40 exposed workers. The data on chromosomal aberrations are given in Table 1. Aberrations produced by the pesticides included gaps, chromatid breaks, isochromatid breaks and exchanges like dicentric, rings and trivalents. The results were compared with those obtained from 30 unexposed healthy controls, who were living in the same communities, as shown in Table 2. When the corresponding data were compared between workers and controls, the workers showed significantly $(p<0.05$ $p<0.01)$ higher rates for numbers of abnormal cells, gaps, chromatid breaks and chromosomal aberrations analyzed separately or combined as compared with the controls in both smokers and non smokers (Table 3 ). In individuals exposed to pesticides, a slight increase (but not significant) $(p>0.05)$ in the frequency of abnormal cell, gaps and chromatid interchanges was observed in the smokers when compared to non smokers.

\section{DISCUSSION}

Extensive studies have been carried out to investigate the genotoxic effects of organophosphorus pesticides [1]. The in vitro and in vivo cytogenetic assay is important for monitoring the genotoxicity of these pesticides $[3,16,18,26]$. In the present study, a cytogenetic investigation was carried out on field workers who were exposed to pesticides and given to the habit of smoking (Table 1). For comparison, studies were also carried out on smokers and non smokers who were not exposed to pesticides (Table 2 ). The breaks induced were mainly of the chromatid type, indicating damage at the $\mathrm{G}_{2}$ phase of the cell cycle (Table 1). Similar effects had been recorded earlier on other mammalian system [27,28].

Statistical analysis revealed that there was a significant increase in chromosomal aberration rate in smokers exposed to pesticides compared to smokers who were not exposed (Table 3). This does not agree with the reported results [29].

As Tables 2 and 3 indicate, the control smokers showed a significant increase in chromosomal aberration rate when compared to non smokers controls. This provides further evidence for the intrinsic mutagenic activity of smoking and agrees with observations reported [30-34].

This study demonstrated a high statistically significant increase in the chromosomal aberrations in the lymphocytes of the exposed smoker and non smoker workers compared with the controls (Table $3)$. These findings agree with the observations made by several authors $[3,16,18-20]$. However, two aspects in the induction of these chromosomal aberrations must be considered. One is that the chromosomal aberration increase could be attributed to the fact that workers were exposed to the pesticides for long periods each year and the level of exposure was enough to produce chromosomal aberrations. The second aspect is that the higher chromosomal aberration frequency might be due to the combined activity of the pesticides. Taking into account that those workers use a large spectrum of pesticides and most of them are not protected by safety measures and, possibly, this kind of exposure is responsible for the observed chromosomal aberration increase. Moreover, this finding is consistent with the observations reported by other authors [18,35-39]. Since the significant increase in chromosomal aberration 
Table 1A. Analysis of structural chromosome aberrations in workers exposed to pesticides

\begin{tabular}{|c|c|c|c|c|c|}
\hline Subject & Age (years) & $\begin{array}{c}\text { Exposed to } \\
\text { Smoking (years) }\end{array}$ & $\begin{array}{c}\text { Exposed to } \\
\text { Pesticides (years) }\end{array}$ & $\begin{array}{l}\text { Number of } \\
\text { Cells Scored }\end{array}$ & $\begin{array}{c}\text { Number of } \\
\text { Abnormal Cells }\end{array}$ \\
\hline \begin{tabular}{|l} 
Smokers \\
1
\end{tabular} & 27 & 4 & 3 & 200 & 6 \\
\hline 2 & 25 & 3 & 2 & 200 & 4 \\
\hline 3 & 24 & 3 & 5 & 200 & 10 \\
\hline 4 & 26 & 2 & 3 & 200 & 4 \\
\hline 5 & 28 & 3 & 4 & 200 & 5 \\
\hline 6 & 26 & 4 & 5 & 200 & 9 \\
\hline 8 & 27 & 5 & 2 & 200 & 3 \\
\hline 9 & 25 & 3 & 4 & 200 & 5 \\
\hline 10 & 27 & 6 & 5 & 200 & 9 \\
\hline 12 & 26 & 3 & 3 & 200 & 4 \\
\hline 13 & 28 & 5 & 4 & 200 & 6 \\
\hline 14 & 27 & 6 & 4 & 200 & 4 \\
\hline 15 & 26 & 5 & 3 & 200 & 5 \\
\hline 17 & 25 & 4 & 2 & 200 & 4 \\
\hline 18 & 27 & 3 & 3 & 200 & 3 \\
\hline 19 & 26 & 2 & 4 & 200 & 8 \\
\hline 21 & 26 & 3 & 5 & 200 & 8 \\
\hline 22 & 27 & 4 & 4 & 200 & 6 \\
\hline 23 & 28 & 2 & 5 & 200 & 9 \\
\hline 24 & 27 & 3 & 2 & 200 & 3 \\
\hline Average & $26.4 \pm 0.24$ & - & - & 4000 & $5.75 \pm 0.05$ \\
\hline $\begin{array}{l}\text { Number of } \\
\text { aberrations/cell }\end{array}$ & - & - & - & - & 0.03 \\
\hline $\begin{array}{l}\text { Non smoker } \\
1\end{array}$ & 27 & 0 & 5 & 200 & 7 \\
\hline 2 & 28 & 0 & 3 & 200 & 4 \\
\hline 3 & 28 & 0 & 2 & 200 & 6 \\
\hline 5 & 26 & 0 & 4 & 200 & 5 \\
\hline 6 & 26 & 0 & 5 & 200 & 7 \\
\hline 7 & 25 & 0 & 4 & 200 & 5 \\
\hline 8 & 27 & 0 & 5 & 200 & 6 \\
\hline 9 & 26 & 0 & 4 & 200 & 5 \\
\hline 10 & 27 & 0 & 4 & 200 & 4 \\
\hline 11 & 28 & 0 & 5 & 200 & 7 \\
\hline 12 & 26 & 0 & 4 & 200 & 7 \\
\hline 14 & 25 & 0 & 3 & 200 & 4 \\
\hline 15 & 27 & 0 & 3 & 200 & 5 \\
\hline 16 & 26 & 0 & 4 & 200 & 5 \\
\hline 17 & 26 & 0 & 4 & 200 & 6 \\
\hline 18 & 29 & 0 & 5 & 200 & 7 \\
\hline 19 & 25 & 0 & 4 & 200 & 6 \\
\hline 20 & 24 & 0 & 3 & 200 & 4 \\
\hline 21 & 25 & 0 & 3 & 200 & 4 \\
\hline 22 & 24 & 0 & 2 & 200 & 3 \\
\hline Average & $26.4 \pm 0.30$ & - & - & 4000 & $3.35 \pm 0.26$ \\
\hline $\begin{array}{l}\text { Number of } \\
\text { aberrations/cell }\end{array}$ & - & - & - & - & 0.03 \\
\hline $\begin{array}{l}\text { Combined } \\
\text { (S+NS) } \\
\text { average }\end{array}$ & $26.3 \pm 0.1$ & - & - & 8000 & $5.35 \pm 0.29$ \\
\hline $\begin{array}{l}\text { Number of } \\
\text { aberrations/cell }\end{array}$ & - & - & - & - & 0.03 \\
\hline
\end{tabular}


Table 1B. Analysis of structural chromosome aberrations in workers exposed to pesticides (continued)

\begin{tabular}{|c|c|c|c|c|c|c|c|c|c|c|c|}
\hline \multirow[b]{2}{*}{ Subject } & \multicolumn{6}{|c|}{ Chromosome Aberrations } & \multirow{2}{*}{$\begin{array}{c}\text { Total } \\
\mathrm{B}^{\prime}+\mathrm{B}^{\prime \prime}+ \\
\mathrm{DIC}^{+}+ \\
\mathrm{DIC}^{-}+\mathrm{RI}\end{array}$} & \multicolumn{3}{|c|}{ Chromosome Interchange } & \multirow{2}{*}{$\begin{array}{c}\begin{array}{c}\text { Total Number } \\
\text { Aberrations/ } \\
100 \text { cells }\end{array} \\
\mathrm{Al}+\mathrm{B} \text { '+B"'+DIC'+DIC } \\
\text { +RI+RB'+RB'B" }\end{array}$} \\
\hline & $\mathrm{Al}$ & $\mathrm{B}^{\prime}$ & B” & $\mathrm{DIC}^{+}$ & $\mathrm{DIC}^{-}$ & RI & & $\mathrm{RB}^{\prime}$ & RB'B”' & Total & \\
\hline $\begin{array}{l}\text { Smokers } \\
1\end{array}$ & 4 & 3 & 2 & 1 & - & - & 6 & - & - & 0 & 5.0 \\
\hline 2 & 3 & 2 & 1 & - & 1 & - & 4 & - & - & 0 & 3.5 \\
\hline 3 & 5 & 3 & 3 & - & - & - & 6 & - & - & 1 & 6.0 \\
\hline 4 & 4 & 3 & 1 & - & 1 & - & 5 & - & - & 0 & 4.5 \\
\hline 5 & 8 & 3 & 2 & - & 1 & - & 6 & 1 & - & 1 & 7.5 \\
\hline 6 & 10 & 4 & 3 & 1 & 1 & 1 & 10 & - & 0 & 1 & 0.0 \\
\hline 8 & 3 & 3 & 1 & - & - & 1 & 5 & - & 0 & 0 & 4.0 \\
\hline 9 & 4 & 3 & 3 & - & 1 & - & 7 & - & 0 & 0 & 5.5 \\
\hline 10 & 8 & 6 & 3 & 1 & - & - & 10 & - & 1 & 1 & 9.5 \\
\hline 12 & 4 & 3 & 2 & - & 1 & - & 6 & - & 0 & 0 & 5.0 \\
\hline 13 & 5 & 3 & 3 & 1 & - & - & 7 & - & 0 & 0 & 6.0 \\
\hline 14 & 4 & 2 & 2 & 1 & - & - & 5 & - & 1 & 1 & 5.0 \\
\hline 15 & 3 & 7 & 2 & - & - & - & 9 & - & - & 0 & 6.0 \\
\hline 17 & 2 & 7 & 1 & - & 1 & - & 9 & - & - & 0 & 5.0 \\
\hline 18 & 2 & 3 & 2 & - & - & - & 5 & - & - & 0 & 3.5 \\
\hline 19 & 4 & 4 & 3 & - & - & - & 7 & - & - & 0 & 5.5 \\
\hline 21 & 9 & 4 & 2 & 1 & 1 & - & 8 & - & - & 0 & 8.5 \\
\hline 22 & 7 & 3 & 3 & - & 3 & 1 & 10 & 1 & - & 1 & 9.0 \\
\hline 23 & 10 & 2 & 2 & - & 1 & 1 & 5 & - & - & 0 & 7.0 \\
\hline 24 & 3 & 4 & 2 & - & - & - & 7 & - & - & 0 & 5.0 \\
\hline Average & $5.10 \pm 0.57$ & 3.70 & 2.16 & 0.30 & 0.60 & 0.20 & 7.10 & 0.10 & 0.15 & 0.25 & 6.10 \\
\hline $\begin{array}{l}\text { Number of } \\
\text { aberrations/cell }\end{array}$ & 0.03 & 0.02 & 0.01 & 0.00 & 0.00 & 0.00 & 0.03 & 0.00 & 0.00 & 0.00 & 0.03 \\
\hline $\begin{array}{l}\text { Non smoker } \\
1\end{array}$ & 7 & 4 & 2 & 1 & - & 8 & - & 0 & 0 & 0 & 7.5 \\
\hline 2 & 4 & 2 & 1 & - & - & - & 3 & - & - & 0 & 3.5 \\
\hline 3 & 3 & 3 & 3 & - & - & - & 6 & - & - & 0 & 4.5 \\
\hline 5 & 4 & 4 & 2 & - & 1 & - & 7 & - & 1 & 1 & 6.0 \\
\hline 6 & 8 & 4 & 1 & - & 1 & - & 6 & 1 & - & 1 & 7.5 \\
\hline 7 & 4 & 3 & 2 & - & - & - & 5 & - & - & 0 & 4.5 \\
\hline 8 & 6 & 2 & 3 & - & 1 & 1 & 7 & - & 1 & 1 & 7.0 \\
\hline 9 & 4 & 3 & 2 & - & 1 & - & 6 & - & - & 0 & 5.0 \\
\hline 10 & 4 & 3 & 1 & - & 1 & - & 5 & - & - & 0 & 4.5 \\
\hline 11 & 7 & 4 & 3 & 1 & - & - & 8 & - & - & 0 & 6.5 \\
\hline 12 & 5 & 2 & 2 & 1 & 1 & - & 5 & - & - & 0 & 5.5 \\
\hline 14 & 4 & 4 & 1 & - & - & - & 5 & - & - & 0 & 4.5 \\
\hline 15 & 3 & 3 & 2 & - & - & 1 & 6 & - & - & 0 & 4.5 \\
\hline 16 & 3 & 3 & 3 & - & - & - & 6 & - & - & 0 & 4.5 \\
\hline 17 & 4 & 2 & 2 & 1 & 1 & - & 6 & - & - & 0 & 5.0 \\
\hline 18 & 5 & 4 & 2 & 1 & 1 & - & 8 & 1 & - & 1 & 7.0 \\
\hline 19 & 3 & 3 & 2 & - & - & - & 5 & - & - & 0 & 4.0 \\
\hline 20 & 3 & 2 & 1 & - & - & - & 3 & - & 1 & 1 & 3.5 \\
\hline 21 & 2 & 3 & 2 & - & - & - & 5 & - & - & 0 & 3.5 \\
\hline 22 & 3 & 3 & 1 & - & 1 & - & 5 & - & - & 0 & 4.0 \\
\hline Average & $5.35 \pm 0.26$ & $3.05 \pm 0.18$ & 1.90 & 0.25 & 0.55 & 0.10 & 5.70 & 0.10 & 0.10 & 0.35 & $5.13 \pm 0.28$ \\
\hline $\begin{array}{l}\text { Number of } \\
\text { aberrations/cell }\end{array}$ & 0.02 & 0.02 & 0.01 & 0.00 & 0.00 & 0.00 & 0.03 & 0.00 & 0.00 & 0.00 & 0.03 \\
\hline $\begin{array}{l}\text { Combined } \\
\text { (S+NS) } \\
\text { average }\end{array}$ & $4.65 \pm 0.34$ & $3.38 \pm 0.18$ & 2.02 & 0.28 & 0.57 & 0.15 & 6.40 & 0.10 & 0.12 & 0.23 & $5.61 \pm 0.27$ \\
\hline $\begin{array}{l}\text { Number of } \\
\text { aberrations/cell }\end{array}$ & 0.02 & 0.02 & 0.00 & 0.00 & 0.03 & 0.00 & 0.00 & 0.00 & 0.00 & 0.00 & 0.03 \\
\hline
\end{tabular}

Al: achromatic lesions (gaps); B': chromatid break; B": isochromatid or chromosome break; RB': chromatid exchange; RB'B": trivalent; $\mathrm{DIC}^{+}$: dicentric chromosome with fragment; $\mathrm{DIC}^{-}$: ducentric chromosome without fragment; RI: ring chromsome; T: total; S: smokers; NS: non smokers.

Abnormal cells with at least one chromosome aberrration. Cells with achromatic lesions only were not scored as abnormal. 
Table 2A. Analysis of structural chromosome aberrations in the control group

\begin{tabular}{|c|c|c|c|c|c|}
\hline Subject & $\begin{array}{c}\text { Age } \\
\text { (years) }\end{array}$ & $\begin{array}{c}\text { Exposed to } \\
\text { Smoking (years) }\end{array}$ & $\begin{array}{c}\text { Exposed to } \\
\text { Pesticides (years) }\end{array}$ & $\begin{array}{c}\text { Number of } \\
\text { Cells Scored }\end{array}$ & $\begin{array}{c}\text { Number of } \\
\text { Abnormal Cells }\end{array}$ \\
\hline $\begin{array}{l}\text { Smokers } \\
1\end{array}$ & 24 & 2 & 0 & 200 & 6 \\
\hline 2 & 26 & 3 & 0 & 200 & 4 \\
\hline 3 & 26 & 3 & 0 & 200 & 8 \\
\hline 4 & 23 & 2 & 0 & 200 & 4 \\
\hline 5 & 27 & 4 & 0 & 200 & 6 \\
\hline 6 & 26 & 5 & 0 & 200 & 8 \\
\hline 7 & 26 & 3 & 0 & 200 & 4 \\
\hline 8 & 24 & 6 & 0 & 200 & 4 \\
\hline 9 & 28 & 5 & 0 & 200 & 4 \\
\hline 10 & 28 & 6 & 0 & 200 & 6 \\
\hline 12 & 25 & 4 & 0 & 200 & 4 \\
\hline 13 & 27 & 4 & 0 & 200 & 4 \\
\hline 14 & 28 & 2 & 0 & 200 & 4 \\
\hline 15 & 28 & 3 & 0 & 200 & 4 \\
\hline 17 & 26 & 4 & 0 & 200 & 6 \\
\hline 18 & 28 & 3 & 0 & 200 & 6 \\
\hline Average & 26.25 & - & - & 3200 & $5.13 \pm 0.36$ \\
\hline $\begin{array}{l}\text { Number of } \\
\text { aberrations/cell }\end{array}$ & - & - & - & - & 0.3 \\
\hline $\begin{array}{l}\text { Non Smokers } \\
1\end{array}$ & 27 & 0 & 0 & 200 & 4 \\
\hline 2 & 28 & 0 & 0 & 200 & 6 \\
\hline 3 & 26 & 0 & 0 & 200 & 6 \\
\hline 4 & 28 & 0 & 0 & 200 & 4 \\
\hline 5 & 25 & 0 & 0 & 200 & 4 \\
\hline 7 & 26 & 0 & 0 & 200 & 6 \\
\hline 8 & 25 & 0 & 0 & 200 & 4 \\
\hline 9 & 24 & 0 & 0 & 200 & 4 \\
\hline 10 & 23 & 0 & 0 & 200 & 4 \\
\hline 11 & 27 & 0 & 0 & 200 & 2 \\
\hline 13 & 26 & 0 & 0 & 200 & 4 \\
\hline 14 & 28 & 0 & 0 & 200 & 4 \\
\hline 15 & 25 & 0 & 0 & 200 & 4 \\
\hline 16 & 28 & 0 & 0 & 200 & 2 \\
\hline Average & 26.14 & - & - & 2800 & $4.14 \pm 0.32$ \\
\hline $\begin{array}{l}\text { Number of aberrations/ } \\
\text { cell }\end{array}$ & - & - & - & - & 0.02 \\
\hline $\begin{array}{l}\text { Combined (S+NS) } \\
\text { average }\end{array}$ & 26.19 & - & - & - & $4.67 \pm 0.24$ \\
\hline $\begin{array}{l}\text { Number of aberrations/ } \\
\text { cell }\end{array}$ & - & - & - & 6000 & 0.02 \\
\hline
\end{tabular}


Table 2B. Analysis of structural chromosome aberrations in the control group (continued)

\begin{tabular}{|c|c|c|c|c|c|c|c|c|c|c|c|}
\hline \multirow[b]{2}{*}{ Subject } & \multicolumn{6}{|c|}{ Chromosome Aberrations } & \multirow{2}{*}{$\begin{array}{c}\text { Total } \\
\\
\mathrm{B}^{\prime}+\mathrm{B}^{\prime \prime+}+ \\
\mathrm{DIC}^{++} \\
\mathrm{DIC}^{-+\mathrm{RI}}\end{array}$} & \multicolumn{3}{|c|}{ Chromosome Interchange } & \multirow{2}{*}{\begin{tabular}{|c|}
$\begin{array}{c}\text { Total Number } \\
\text { Aberrations/ } \\
100 \text { cells }\end{array}$ \\
AI+B'+B"+DIC \\
+DIC'+RI+RB'+ \\
RB'B" \\
\end{tabular}} \\
\hline & $\mathrm{Al}$ & $\mathrm{B}^{\prime}$ & B" & $\mathrm{DIC}^{+}$ & $\mathrm{DIC}^{-}$ & RI & & $\mathrm{RB}^{\prime}$ & RB'B" & Total & \\
\hline $\begin{array}{l}\text { Smokers } \\
1\end{array}$ & 4 & 4 & 1 & 1 & 0 & 0 & 6 & 0 & 0 & 0 & 5.0 \\
\hline 2 & 2 & 2 & 1 & 0 & 0 & 0 & 3 & 0 & 0 & 0 & 2.5 \\
\hline 3 & 4 & 6 & 3 & 1 & 0 & 0 & 10 & 0 & 0 & 0 & 7.0 \\
\hline 4 & 4 & 2 & 2 & 0 & 0 & 0 & 4 & 0 & 0 & 0 & 4.0 \\
\hline 5 & 2 & 2 & 2 & 0 & 1 & 0 & 5 & 0 & 0 & 0 & 3.5 \\
\hline 6 & 4 & 4 & 0 & 0 & 1 & 1 & 6 & 0 & 0 & 0 & 5.0 \\
\hline 7 & 4 & 2 & 2 & 0 & 0 & 0 & 4 & 1 & 0 & 1 & 4.5 \\
\hline 8 & 4 & 4 & 0 & 0 & 0 & 1 & 5 & 0 & 0 & 0 & 4.5 \\
\hline 9 & 6 & 5 & 2 & 0 & 0 & 0 & 7 & 1 & 0 & 1 & 7.0 \\
\hline 10 & 2 & 5 & 2 & 1 & 0 & 0 & 8 & 0 & 0 & 0 & 5.0 \\
\hline 12 & 3 & 2 & 0 & 0 & 1 & 0 & 3 & 0 & 0 & 0 & 3.0 \\
\hline 13 & 3 & 4 & 2 & 0 & 0 & 0 & 6 & 0 & 0 & 0 & 4.5 \\
\hline 14 & 2 & 2 & 1 & 1 & 1 & 0 & 5 & 0 & 0 & 0 & 3.5 \\
\hline 15 & 2 & 2 & 1 & 0 & 0 & 0 & 3 & 0 & 0 & 0 & 2.5 \\
\hline 17 & 4 & 4 & 2 & 1 & 1 & 0 & 8 & 0 & 0 & 0 & 6.0 \\
\hline 18 & 4 & 4 & 4 & 0 & 0 & 0 & 8 & 0 & 0 & 0 & 6.0 \\
\hline Average & $3.32 \pm 0.27$ & $3.38 \pm 0.34$ & $1.56 \pm 0.30$ & 0.25 & 0.31 & 0.13 & $5.68 \pm 0.52$ & 0.13 & 0.00 & $0.12 \pm 0.08$ & $4.59 \pm 0.35$ \\
\hline $\begin{array}{l}\text { Number of } \\
\text { aberrations/cell }\end{array}$ & 0.02 & 0.02 & 0.01 & 0.00 & 0.00 & 0.00 & - & 0.00 & 0.00 & 0.00 & - \\
\hline $\begin{array}{l}\text { Non Smokers } \\
1\end{array}$ & 1 & 2 & 0 & 0 & 0 & 0 & 2 & 0 & 0 & 0 & 1.5 \\
\hline 2 & 2 & 2 & 0 & 0 & 0 & 4 & 0 & 0 & 0 & 0 & 2.5 \\
\hline 3 & 2 & 3 & 1 & 0 & 0 & 0 & 4 & 0 & 0 & 0 & 3.0 \\
\hline 4 & 2 & 2 & 2 & 0 & 0 & 0 & 4 & 0 & 0 & 0 & 3.0 \\
\hline 5 & 1 & 2 & 0 & 0 & 0 & 0 & 2 & 0 & 0 & 0 & 1.5 \\
\hline 7 & 1 & 3 & 1 & 0 & 0 & 0 & 4 & 0 & 0 & 0 & 2.5 \\
\hline 8 & 0 & 2 & 0 & 0 & 0 & 0 & 2 & 0 & 0 & 0 & 1.0 \\
\hline 9 & 2 & 2 & 1 & 0 & 0 & 0 & 3 & 0 & 0 & 0 & 2.5 \\
\hline 10 & 1 & 2 & 1 & 0 & 0 & 0 & 3 & 0 & 0 & 0 & 2.0 \\
\hline 11 & 1 & 0 & 0 & 0 & 0 & 0 & 0 & 0 & 0 & 0 & 0.5 \\
\hline 13 & 2 & 2 & 1 & 0 & 0 & 0 & 3 & 0 & 0 & 0 & 2.5 \\
\hline 14 & 2 & 2 & 2 & 0 & 0 & 0 & 4 & 0 & 0 & 0 & 3.0 \\
\hline 15 & 0 & 2 & 1 & 0 & 0 & 0 & 3 & 0 & 0 & 0 & 1.5 \\
\hline 16 & 2 & 0 & 1 & 0 & 0 & 0 & 1 & 0 & 0 & 0 & 1.5 \\
\hline Average & $1.29 \pm 0.19$ & $1.86 \pm 0.23$ & $0.93 \pm 0.19$ & 0.00 & 0.00 & 0.00 & $2.78 \pm 0.71$ & 0.00 & 0.00 & 0.00 & $2.04 \pm 0.21$ \\
\hline $\begin{array}{l}\text { Number of } \\
\text { aberrations/cell }\end{array}$ & 0.01 & 0.01 & 0.00 & 0.00 & 0.00 & 0.00 & 0.01 & 0.00 & 0.00 & 0.00 & 0.01 \\
\hline $\begin{array}{l}\text { Combined (S+NS) } \\
\text { average }\end{array}$ & $2.33 \pm 0.18$ & $2.66 \pm 0.25$ & $1.30 \pm 0.17$ & 0.13 & 0.15 & 0.07 & $4.33 \pm 0.41$ & 0.07 & 0.00 & $0.06 \pm 0.11$ & $3.32 \pm 0.23$ \\
\hline $\begin{array}{l}\text { Number of } \\
\text { aberrations/cell }\end{array}$ & 0.01 & 0.01 & 0.01 & 0.00 & 0.00 & 0.00 & 0.02 & 0.00 & 0.00 & 0.00 & 0.02 \\
\hline
\end{tabular}

Al: achromatic lesions (gaps); B': chromatid break; B": isochromatid or chromosome break; RB': chromatid exchange; RB'B': trivalent; DIC ${ }^{+}$: dicentric chromosome with fragment; DIC ${ }^{-}$: ducentric chromosome without fragment; RI: ring chromsome; T: total; S: smokers; NS: non smokers.

Abnormal cells with at least one chromosome aberrration. Cells with achromatic lesions only were not scored as abnormal. 
Table 3. Statistical analysis of the means

\begin{tabular}{|c|c|c|c|}
\hline Means & Comparison & $t$ Value & Probability \\
\hline $\begin{array}{l}\text { I. Workers } \\
\text { Number of abnormal cells } \\
\text { A chromatic lesion (gap) } \\
\text { Chromatid break (B') } \\
\text { Chromosome aberrations (B'+B"+DIC }+ \text { DIC }+ \text { R1) } \\
\text { Chromatid interchange (RB'+RB'B') } \\
\text { Total number of aberrations/100 cells }\end{array}$ & $\begin{array}{l}\mathrm{S} v s . \mathrm{NS} \\
\mathrm{S} v s . \mathrm{NS} \\
\mathrm{S} v s . \mathrm{NS} \\
\mathrm{S} v s . \mathrm{NS} \\
\mathrm{S} v s . \mathrm{NS} \\
\mathrm{S} v s . \mathrm{NS}\end{array}$ & $\begin{array}{l}0.2460 \\
1.3513 \\
3.1553 \\
2.5706 \\
0.4274 \\
2.1320\end{array}$ & $\begin{array}{l}p>0.05 \\
p>0.05 \\
p<0.01 \\
p<0.05 \\
p>0.05 \\
p<0.05\end{array}$ \\
\hline $\begin{array}{l}\text { II. Controls } \\
\text { Number of abnormal cells } \\
\text { A chromatic lesion (gap) } \\
\text { Chromatid break (B') } \\
\text { Chromosome aberrations (B'+B"+DIC }+ \text { DIC-+R1) } \\
\text { Chromatid interchange (RB'+RB'B") } \\
\text { Total number of aberrations/100 cells }\end{array}$ & $\begin{array}{l}\text { S vs. NS } \\
\text { S vs. NS } \\
\text { S vs. NS } \\
\text { S vs. NS } \\
\text { S vs. NS } \\
\text { S vs. NS }\end{array}$ & $\begin{array}{l}2.0008 \\
6.8104 \\
3.5899 \\
3.3437 \\
1.3888 \\
6.4399\end{array}$ & $\begin{array}{l}p<0.05 \\
p<0.01 \\
p<0.01 \\
p<0.01 \\
p>0.05 \\
p<0.01\end{array}$ \\
\hline $\begin{array}{l}\text { III. Combined (S+NS) } \\
\text { Number of abnormal cells } \\
\text { A chromatic lesion (gap) } \\
\text { Chromatid break (B') } \\
\text { Chromosome aberrations (B'+B"+DIC }+ \text { DIC }+ \text { R1) } \\
\text { Chromatid interchange (RB'+RB'B') } \\
\text { Total number of aberrations/100 cells }\end{array}$ & $\begin{array}{l}\text { W vs. NS } \\
\text { W vs. NS } \\
\text { W vs. NS } \\
\text { W vs. NS } \\
\text { W vs. NS } \\
\text { W vs. NS }\end{array}$ & $\begin{array}{l}2.2055 \\
5.3050 \\
2.8356 \\
2.8706 \\
0.9571 \\
6.2194\end{array}$ & $\begin{array}{l}p<0.05 \\
p<0.01 \\
p<0.05 \\
p<0.01 \\
p>0.05 \\
p<0.01\end{array}$ \\
\hline $\begin{array}{l}\text { IV. Smokers } \\
\text { Number of abnormal cells } \\
\text { A chromatic lesion (gap) } \\
\text { Chromatid break (B') } \\
\text { Chromosome aberrations (B'+B"+DIC }+ \text { DIC-+R1) } \\
\text { Chromatid interchange (RB'+RB'B") } \\
\text { Total number of aberrations/100 cells }\end{array}$ & $\begin{array}{l}\text { W vs. NS } \\
\text { W vs. NS } \\
\text { W vs. NS } \\
\text { W vs. NS } \\
\text { W vs. NS } \\
\text { W vs. NS }\end{array}$ & $\begin{array}{l}0.9934 \\
2.5145 \\
0.6721 \\
2.0910 \\
0.7488 \\
2.5989\end{array}$ & $\begin{array}{l}p>0.05 \\
p<0.05 \\
p>0.05 \\
p<0.05 \\
p>0.05 \\
p<0.01\end{array}$ \\
\hline $\begin{array}{l}\text { V. Non Smokers } \\
\text { Number of abnormal cells } \\
\text { A chromatic lesion (gap) } \\
\text { Chromatid break (B') } \\
\text { Chromosome aberrations (B'+B'+DIC }+ \text { DIC }+ \text { R1) } \\
\text { Chromatid interchange (RB'+RB'B') } \\
\text { Total number of aberrations/100 cells }\end{array}$ & $\begin{array}{l}\text { W vs. NS } \\
\text { W vs. NS } \\
\text { W vs. NS } \\
\text { W vs. NS } \\
\text { W vs. NS } \\
\text { W vs. NS }\end{array}$ & $\begin{array}{l}2.9533 \\
6.8341 \\
4.1975 \\
4.0813 \\
2.2222 \\
7.7657\end{array}$ & $\begin{array}{l}p<0.01 \\
p<0.01 \\
p<0.01 \\
p<0.01 \\
p<0.05 \\
p<0.01\end{array}$ \\
\hline
\end{tabular}

S: smokers; NS: non smokers; W: workers; C: controls.

in the present study could be due to the fact that the workers were exposed to two pesticides, a further study should include a cytogenetic evaluation of individual pesticide.

\section{CONCLUSIONS}

This first cytogenetic study performed in Jordan revealed a highly significant increase in the number of chromosomal aberrations in lymphocytes of agricultural workers, smokers and non smokers, exposed to malathion and chlorpyrifos. Therefore, it is recommended that precautionary measures should be taken and that smoking should be avoided while spraying.

The exposed group needs further biological tests and follow-up to identify other potential factors leading to later malignancy development. A new environmental policy with a rational strategy 
is needed to reduce the contamination and genetic risks to these agricultural workers. These considerations may yield greater insights, greater awareness, and modified public policies, and increase activity to mitigate these adverse effects.

\section{REFERENCES}

1. Wild D. Mutagenicity studies on organophosphorus insecticides. Mutation Res 1975; 32: 133150 .

2. Strokum H. Pesticides suspected of causing infertility. Am J Indust Med 1983; 24: 587-592.

3. Rupa DS, Rao PVL, Reddi OS. In vitro effect of monocrophos on human lymphocyte cultures. Bull Enviro Contam Toxicol 1988; 41: 737-741.

4. Watson AD, Berliner JA, Hama SY, La Du BN, Faull KF, Fogehnan AM, Navab M. Protective effect of high density lipoprotein associated para oxonase: inhibition of the biological activity of minimally oxidized low-density lipoprotein. J Clin Invest 1995; 96: 2882-2891.

5. Timchalk C, Poet TS, Kousba AA. Age-dependent pharmacokinetic and pharmacodynamic response in preweanling rats following oral exposure to the organophosphorus insecticide chlorpyrifos. Toxicology 2006; 220: 13-25

6. WHO. (2007) WHO Specifications and Evaluations for Public Health Pesticides. Chlorpyrifos. World Health Organization, p.17. www.who.int/entity/whopes/quality/chlorpyrifos-WHO-specs- Aug2007.pdf.

7. Mulanovich GS, Lescano AG, Gonzaga VE, Blazes DL. Occupational Health in Developing World: A Role for the Medical Research Community. Occup Environ Med 2007; 49: 1184-1188.

8. Rabello MN, Becak W, De Almedia WF, Pigati P, Ungaro MT, Murata T, Pereira CAB. Cytogenetic study on individuals occupationally exposed to DDT. Mutation Res 1975; 28: 449-454.

9. Chen HH, Siriani JL, Huang CC. Sister-chromatid exchanges and cell-cycle delay in Chinese hamster V-79 cells treated with 9 organophosphorus compounds ( 8 pesticides and 1 defoliant). Mutation Res 1982; 103: 307-313.

10. Wang TC, Lee TC, Lin MF, Lin SY. Induction of sister-chromatid exchanges by pesticides in primary rat tracheal epithelial cells and Chinese hamster ovary cells. Mutation Res 1987; 188: 311-
321.

11. Salvadori DMF, Ribeiro LR, Pereira CAB, Becak W. Cytogenetic effects of malathion insecticide on somatic and germ cells of mice. Mutation Res 1988; 204: 283-287.

12. Lessa JMM, Becak W, Rabello MN, Pereira CAB, Ungaro MT. Cytogenetic study of DDT on human lymphocytes in vitro. Mutation Res 1976; 40: 131-138.

13. Nicholas HA, Vienne M, Berghe HVD. Induction of sister chromatid exchanges in cultured human cells by an organophosphorus insecticide malathion. Mutation Res 1979; 67: 167-172.

14. Chen HH, Hsueh JI, Sirianni SR, Huang CC. Induction of sister-chromatid exchanges and cell cycle delay in cultured mammalian cells treated with eight organophosphorus pesticides. Mutation Res 1981; 88: 307-316.

15. Sobti RC, Krishan A, Pfaffenberger CD. Cytokinetic and cytogenetic effects of some agricultural chemicals on human lymphoid cells in vitro: organophosphate. Mutation Res 1982; 102: 89-102.

16. Balaji M, Sasikala K. Chromosome damage occurs to human blood cells. Mutation Res 1993; 301: 13-17.

17. Hoerauf P, Spaccek A, Brunnberg L, Nussa M. Genetic damage in operating room personnel exposed to isoflurane and nitrous oxide. Occup Environ Med 1999; 56: 433-437.

18. Padly A, Puskas N, Vincze K, Hadhazi M. Cytogenetic studies on rural population exposed to pesticides. Mutation Res 1987; 187: 127-132.

19. Rita P, Reddy PP, Reddy SV. Monitoring of workers occupationally exposed to pesticides in grape gardens of Andhra Pradesh. Environ Res 1987; 44: $1-5$.

20. Dulout FN, Pastori MC, Olivero OA, Gonzalez Cid M, Loria D, Matos E, Sobel N, de bujan EC, Albino N. Sister-chromatid exchanges and chromosome aberrations in a population exposed to pesticides. Mutation Res 1985; 143: 237-244.

21. Feretti D, Zerbini I, Zani C, Ceretti E, Moretti M, Monarca S. Allium cepa chromosome aberration and micronucleus tests applied to study genotoxicity of extracts. Food Additives and Contaminants 2007; 24: 561-572.

22. Stocco R, Becak W, Gaeta R, Rabello-Gay MN. Cytogenetic studies of workers exposed to methyl parathion. Mutation Res 1982; 103: 71-76. 
23. Linnainma K. Sister chromatid exchanges among workers occupationally exposed to phenoxyacid herbicides 2,4-D and MCPA. Teratogen Carcinogen Mutagen 1983; 3: 269-279.

24. Hungerford D A. Leukocyte cultured from small inocula of whole blood and the preparation of metaphase chromosomes by treatment with hypotonic KCl. Stain Technol 1965; 40: 333-338.

25. Buckton KC, Evans HJ (eds). Methods for the analysis of human chromosome aberrations. Geneva: WHO Report, 1973.

26. Ray VA. Approaches to the genetic evaluation of agricultural chemicals. In: Fleck RA, Hollaender H, Eds. Genetic Toxicology. An Agricultural Perspective. New York: Plenum. 1982; 45-46.

27. Singh CBP, Sharma A, Talukder E. Effects of chromium on cellular systems in animals. Nucleus 1990; 33: 84-106.

28. Gauglhofer J, Bianchi V. Chromium. In: Meriun E, Ed. Metals and Their Compounds in the Environment. Weinheim: VCH. 1991; 853-878.

29. Mirkova ET, Lalchev SG. The genetic toxicity of the human carcinogens benzidine and benzidine based dyes: chromosomal analysis in exposed workers. Mutation and Environment 1990; Part c, 397-405.

30. Husgalvel-Pursianin K, Maki-Poakkanen J, Worppa H, Sorsa M. Smoking and sister chromatid exchange. Hereditas 1980; 92: 247-250.

31. Obe G, Vogt HJ, Modle S, Fahning A, Heller WD. Double-blind study on the effect of cigarette smoking on the chromosomes of human peripheral blood lymphocytes in vivo. Mutation Res 1982; 92 : 309-319.

32. Vijayalaxmi EHJ. In vivo and in vitro effects of cigarette smoke on chromosomal damage and sister chromatid exchange in human peripheral lymphocytes. Mutation Res 1982; 92: 321-332.

33. Wulf HC, Husun B, Niebuhr E. Cessation of smoking enhances sister chromatid exchanges in lymphocytes. Hereditas 1985; 102: 195-198.

34. Omari YI. The frequency of chromosome aberrations in lymphocytes of young smoking and non-smoking Jordanian males. Broteria Genetica 1996; XV11(xc11): 57-63.

35. Crossen PE, Morgan WF, Horan JJ, Stewart J. Cytogenetic study of pesticide and heritable sprayers. NZ Med J 1978; 88: 192-195.

36. Nehez M, Berensci G, Paldy A, Selypes A, Czeizel E, Szentesi J, Csanko I, Levay K, Maurer J, Nagy E. Data on the chromosome examination of workers exposed to pesticide. Regul Toxicol Pharmacol 1981; 1: 116-119.

37. Shabtai E, Bichacho S, Halbrecht I. Cytogenetic observations in infertile men working with insecticidal compounds. Acta Genet Med Gemellol 1979; 26: 51-56.

38. Voinjanskya AB, Vasilos AF. Level of chromosome aberrations in agricultural workers. Gid Truda 1981; 12: 47-48.

39. Pilinskaya MA. Importance of cytogenetic investigations of professional contacts for hygienic evaluation of pesticides. Gig Truda Prof Zabol 1982; 4: 28-32. 